\title{
Concordance of triage performed with emergency severity index in the emergency departments of Rasoul Akram and Haft Tir hospitals
}

\author{
Nader Tavakoli ${ }^{\circledR}$, Saeed Abbasi ${ }^{2}$, Afrooz Tayebi ${ }^{3^{*}}$ \\ ${ }^{1}$ Emergency Medicine Department, Trauma and Injury Research Center, Iran University of Medical Sciences, Tehran, Iran \\ ${ }^{2}$ Emergency Medicine Management Research Center, Iran University of Medical Sciences, Tehran, Iran \\ ${ }^{3}$ Emergency Physician, Iran University of Medical Sciences, Tehran, Iran
}

\section{Correspondence to:}

Afrooz Tayebi,

Email:

dr.afrooz.tayebi@gmail.com, Tayebi.a@iums.ac.ir

Received: 4 August 2020 Accepted: 4 Oct. 2020 ePublished: 29 Oct. 2020

Keywords: Triage, Emergency severity index, Emergency department, Treatment outcome

\begin{abstract}
Introduction: Triage is prioritizing patients by disease severity in the shortest possible time. Proper triage increases the quality of patient care services, increases patient's satisfaction, decreases patient waiting time, decreases mortality, and enhances emergency department efficiency.

Objectives: The aim of this study was to evaluate the concordance of triage performed by nurses with ESI standard, at Rasoul Akram and Haft Tir hospitals, which are the most important educational centers of Iran University of Medical Sciences.

Patients and Methods: This is a cross-sectional design study done in 2019. The study population is patients referred to Rasoul Akram and Haft Tir hospitals. A sample of 800 patients was selected. In this study, we determined how triage level were determined and compared with triaging by emergency medicine assistant. Data were analyzed by SPSS 23.

Results: Out of 800 samples, the highest triage level was related to level three $(79.1 \%)$ and levels two, four and one were in the next levels. $13.5 \%$ of the cases were not properly triaged according to the ESI standard and the adaptation coefficient (kappa) between the triage level specified in the patient file with the emergency severity index (ESI) triage level between physician and nurse was $58 \%$, which was a significant difference $(P<0.001)$. The relationship between correct triage leveling and patients' complaints was significant $(P<0.001)$.

Conclusion: Nurses triage education about appropriate triage and ESI tool should be one of the most urgent topics and priorities of the studied hospitals. Over triage causes resources wasted and under triage may harm the patient's health.
\end{abstract}

Citation: Tavakoli
N, Abbasi S, Tayebi
A. Concordance of
triage performed with
emergency severity
index in the emergency
departments of Rasoul
Akram and Haft
Tir hospitals. J Prev
Epidemiol. 2020;5(1):e11.
doi: $10.34172 /$
jpe.2020.11.

\section{Introduction}

Emergency situations are high-risk situations in which the physical or mental condition of a person is suddenly affected and he/she require prompt, basic, and appropriate action as needed $(1,2)$. Triage is the patient prioritization process based on the severity of the illness in order to perform the best treatment in the shortest possible time, which is on the basis of the patient need in the emergency department $(3,4)$. The purpose of triage in the emergency department is to answer the question, "At this time and for particular patient, what priority should be given in relation to other patients in the emergency department?"(5). Triage is performed prior to admission and patients in need of cardiopulmonary resuscitation are in the first priority. Triage is performed by a nurse or general practitioner who has at least one-year experience in

\begin{abstract}
Key point
Triage is prioritizing patients by disease severity in the shortest possible time. In this study, evaluated the concordance of triage performed by nurses with emergency severity index (ESI), at Rasoul Akram and Haft Tir hospitals. Out of 800 samples, $13.5 \%$ of the cases were not properly triaged according to the ESI standard and the adaptation coefficient (kappa) between the triage level specified in the patient file with the ESI triage level between physician and nurse was $58 \%$, which was a significant difference $(P<00.001)$. The relationship between correct triage leveling and patients' complaints was significant $(P<00.001)$. Nurses triage education about appropriate triage and ESI tool should be one of the most urgent topics and priorities of the studied hospitals.
\end{abstract}

the intensive care unit and four years in the emergency department. In the study of Safari et al discussing a variety of world-wide triage methods, one of the most commonly used tools was ESI standards (6). The ESI

Copyright ( 2020 The Author(s); Published by Society of Diabetic Nephropathy Prevention. This is an open-access article distributed under the terms of the Creative Commons Attribution License (http://creativecommons.org/licenses/by/4.0), which permits unrestricted use, distribution, and reproduction in any medium, provided the original work is properly cited. 
standard is a five-level hospital triage tool. In addition to specifying which patient should be visited first, it indirectly determines what level of facilities and resources are needed to serve the patient. In this system, patients are divided into five immediate to delayed priorities based on the severity of the illness and the severity of the disease. This tool is a useful and promising tool that can be used in all urban and rural emergencies and public and university hospitals (7).

\section{Objectives}

Various studies have shown that using the ESI standard improves emergency department performance by reducing patient triage time and it is also a valid and reliable tool for triaging in emergencies department (8-10). Triage is done by a nurse in educational hospitals in Iran. Therefore, in this study, we evaluate the concordance of triage performed by nurses with ESI standard, at Rasoul Akram and Haft Tir hospitals, which are the most important educational centers of Iran University of Medical Sciences.

\section{Patients and Methods}

\section{Study design}

This is a cross-sectional descriptive study conducted in 2019. The study sample was patients referred to Rasoul Akram and Haft Tir hospitals. In this study, the sample of 800 patients was selected from 400 of Rasoul Akram hospital and 400 from Haft Tir hospital. The Sample was selected from the available/easy sampling method during the study period and since the triage has been done by the nurse in the hospital, the accuracy of triage level performed according to ESI standard was reviewed by the emergency medicine assistant.

\section{Ethical issues}

Human rights were respected in accordance with the Helsinki Declaration 1975, as revised in 1983. The ethical committee of Iran University of Medical Sciences (Ethical code\# IR.IUMS.FMD.REC.1397.170) approved the study. The informed consent was taken from the patients as well as from parents and first relatives. This paper was extracted from the specialty medical thesis in emergency medicine of Afrooz Tayebi (Thesis\#793) in Iran University of Medical Sciences Faculty of Medical (Emergency Department).

\section{Statistical analysis}

The data were analyzed by IBM SPSS 23 statistical package (SPSS Inc, Chicago, IL, USA). Results were analyzed using descriptive statistics methods, frequencies, cumulative frequency percentage and charts. To analyze the data, kappa test, independent $t$ test, chi-square test, and oneway ANOVA were conducted. To report results, 5\% was considered as significance level.

\section{Results}

Of the 800 patients studied, 481 (60\%) were male and 319 (40\%) were female. Table 1 shows information on triage levels performed in 800 cases by nurses in the studied hospitals. The highest frequency of triage level was related to level three (79\%) and then levels two, four and one were in the next grade. The rate of concordance between the levels of actual triage specified in the patient records with the level of ESI triage standard was assessed. According to Table 1 , it is clear that the kappa value is $58 \%$, indicating a significant difference $(P<0.001)$. Therefore, according to this value, the concordance is moderate and the observed level is statistically different with the expected level. Finally, of the 800 cases, 107 were not properly triaged.

In Table 2, the difference in vital signs difference between the patients with correct triage and incorrect triage. The age of patients in the group with incorrect triage was higher than that of the correct group and this difference was significant $(P=0.003)$. In addition, respiratory rate was significantly different between the two groups $(P=$ 0.008). Additionally, pulse rate was higher in the incorrect triage patients and lower oxygen saturation level compared to the correct triage patients.

Table 3 shows the relationship between correct triage level and type of complaint and referral. In people with chest pain, malaise, and multiple traumas incorrect triage level is higher. In patients with chest pain $17.1 \%$, malaise $16.9 \%$ and in patients with multiple trauma $16.8 \%$. Finally, the relationship between correct triage level and the cause of the complaint was significant $(P<0.001)$.

The results of Table 4 show the relation of triage level with treatment outcome (discharge, hospitalization, death, and referral). Incorrect level of triage was more in death and referral layers. Incorrect level of triage was $25 \%$ in death layer and $18.7 \%$ in referral layer. This difference in

Table 1. Triage concordance with ESI

\begin{tabular}{|c|c|c|c|c|c|}
\hline \multirow{2}{*}{ Triage level } & \multicolumn{4}{|c|}{ ESI level } & \multirow{2}{*}{ Total } \\
\hline & 1 & 2 & 3 & 4 & \\
\hline 1 & $12(63.2)$ & $0(0)$ & $2(0.3)$ & $0(0)$ & $14(1.8)$ \\
\hline 2 & $6(31.6)$ & 69 (70.4) & $59(8.8)$ & $0(0)$ & $134(16.8)$ \\
\hline 3 & $1(5.3)$ & $29(29.62)$ & $600(89.6)$ & $2(15.4)$ & $632(79)$ \\
\hline 4 & $0(0)$ & $0(0)$ & $8(1.2)$ & $11(84.6)$ & $19(2.4)$ \\
\hline Total & 19 (100) & $98(100)$ & $670(100)$ & 13 (100) & 800 (100) \\
\hline
\end{tabular}


Table 2. Mean difference in vital signs

\begin{tabular}{|c|c|c|c|c|c|}
\hline Variable & Triage group & Mean & Standard deviation & $T$ & $P$ value \\
\hline \multirow{2}{*}{ Age } & Correct & 37.25 & 20.38 & \multirow{2}{*}{-3.02} & \multirow{2}{*}{0.003} \\
\hline & Incorrect & 43.72 & 21.35 & & \\
\hline \multirow{2}{*}{ Blood pressure } & Correct & 120.46 & 17.52 & \multirow{2}{*}{-0.91} & \multirow{2}{*}{0.37} \\
\hline & Incorrect & 122.55 & 21.86 & & \\
\hline \multirow{2}{*}{ Pulse rate } & Correct & 81.73 & 9.32 & \multirow{2}{*}{-1.61} & \multirow{2}{*}{0.11} \\
\hline & Incorrect & 83.78 & 21.15 & & \\
\hline \multirow{2}{*}{ Breath rate } & Correct & 16.24 & 1.92 & \multirow{2}{*}{-2.67} & \multirow{2}{*}{0.008} \\
\hline & Incorrect & 16.81 & 2.27 & & \\
\hline \multirow{2}{*}{ Oxygen saturation level } & Correct & 96.13 & 2.16 & \multirow{2}{*}{-1.87} & \multirow{2}{*}{0.06} \\
\hline & Incorrect & 95.51 & 3.18 & & \\
\hline \multirow{2}{*}{ Body temperature } & Correct & 37.24 & 0.23 & \multirow{2}{*}{0.97} & \multirow{2}{*}{0.34} \\
\hline & Incorrect & 37.21 & 0.24 & & \\
\hline
\end{tabular}

the layers indicates no significant difference $(P=0.41)$. The accuracy of triage level in Haft Tir hospital is more than Rasoul Akram hospital $(P<0.001)$.

\section{Discussion}

In the present study, as the results showed, 800 triaged patients' documents were assessed by the Emergency Medicine Assistant. The majority of the sample were male (60\%). Rasoul Akram and Haft Tir hospitals are the trauma centers in Tehran city and naturally, as men are more exposed to trauma, so they have a larger sample size. The results of Table 1 showed that $79.1 \%$ of patients were in third level of triage. In the Dehnadi et al study, $8 \%$ of patients had acute, $52 \%$ emergency, and $38 \%$ nonemergency conditions, indicating that the majority of patients were in semi-acute conditions (level three) (11).

Table 3. Relationship between triage grouping and cause of complaint

\begin{tabular}{lccc}
\hline \multirow{2}{*}{ Cause of complaint } & \multicolumn{2}{c}{ Triage level } & \multirow{2}{*}{ Total } \\
\cline { 2 - 3 } & Correct & Incorrect & \\
\hline Direct trauma & $297(92.2)$ & $25(7.8)$ & 322 \\
\hline Malaise & $108(83.1)$ & $22(16.9)$ & 130 \\
\hline Multiple trauma & $104(83.2)$ & $21(16.8)$ & 125 \\
\hline Valve trauma & $62(91.2)$ & $6(8.8)$ & 68 \\
\hline Stomach ache & $44(88)$ & $6(12)$ & 50 \\
\hline Chest pain & $29(82.9)$ & $6(17.1)$ & 35 \\
\hline Others & $49(70)$ & $21(30)$ & 70 \\
\hline Total & $693(86.7)$ & $107(13.3)$ & 800 \\
\hline
\end{tabular}

Table 4. Relationship between triage and outcome type

\begin{tabular}{lccc}
\hline \multirow{2}{*}{ Cause of complaint } & \multicolumn{2}{c}{ Triage level } & \multirow{2}{*}{ Total } \\
\cline { 2 - 3 } & Correct & Incorrect & \\
\hline Discharge & $621(87.1)$ & $92(12.9)$ & 713 \\
\hline Hospitalization & $44(86.3)$ & $7(13.7)$ & 51 \\
Death & $15(0.75)$ & $5(0.25)$ & 20 \\
Referral & $62(81.3)$ & $3(18.7)$ & 16 \\
\hline Total & $693(86.7)$ & $107(13.3)$ & 800 \\
\hline
\end{tabular}

In the Kamrani and colleagues' study, most patients were triaged at level three (12). In the Nicole study, patients were triaged according to ESI at level one $0.25 \%$, level two $9.4 \%$, level three $37 \%$, level four $32 \%$ and level five $\% 21$ (10). Incorrect triage was more common in patients with chest pain (17.1\%), malaise (16.9\%) and multiple trauma (16.8\%), and this relationship was significant in the present study. Kappa coefficient was 58\% in this study, 107 out of 800 cases were not properly triaged, indicating a significant difference between triage level of nurse and emergency medicine assistant. This adjustment showed the moderate concordance of nurses' triage with ESI standard. Incorrect triages in Rasoul Akram hospital were more than Haft Tir hospital. While both are trauma center hospitals, Rasoul Akram hospital is larger and more comprehensive hospital than Haft Tir hospital and the variety of patients may cause inaccurate triage but Haft Tir hospital has mostly trauma patients and there is less variety of patient's referred. On the other hand, this difference may be due to the higher level of education and more appropriate performance of the nurses at Haft Tir hospital than Rasool Akram hospital. In the study by Kariman et al, The coefficient of agreement (kappa) between physician-nurse triage levels was $87 \%$, which was higher than our study (13). In the Esmailian and colleagues' study, the kappa coefficient between physician and nurse was $94 \%$, in Jafari's study; $82 \%$, in Rahmani and colleagues' study; $65 \%$, in Nicole's study; 92\%, in Rutschmann and colleagues' study; $84.1 \%$ and in Savatmongkorngul and colleagues' study, the kappa coefficient between physician and nurse was $69 \%$. All kappa coefficients were higher than our study (1418). In study by Mirhaghi et al, which compared nurses and physician's triage with the gold standard, triage of nurses, physicians and experts was 0.54, 0.45 and 0.81 , respectively which were in line with gold standard (19). In a similar study by Seyedhosseini-Davarani et al, $81 \%$ of physicians and $96 \%$ of nurses were in compliance with the gold standard (20). In the study by Rahmani et al, as in our study, there was a significant relationship between physician and nurse triage. However, in studies of triage concordant between the nurse and the pre-hospital 
technician, the kappa coefficient was lower than our study $(21,22)$. Vital signs in the respiratory and oxygen saturation indices were significant in our study, meaning that patients in the incorrect triage had significantly higher respiratory rate and lower oxygen saturation than those in the correct triage group. There was no significant difference in other vital signs. However, in the study by Rutschmann et al, the relationship between vital signs and triage level was significant (17). Given the sophisticated equipment available today in hospitals' emergency care, training in the correct measuring and registration of vital signs can greatly assist in proper triage according to the ESI. In the Ng and colleagues' study, triage training according to ESI improves nursing performance in correct triaging (9); also, the relationship between age and triage level was significant and age was higher in the incorrect triage patients than in the correct triage, indicating that age was a factor affecting the nurse's mind in the triage. This finding was different from the study by Kamrani et al, who found no significant relationship between age and triage level (12). Around $89.1 \%$ of the patients were discharged from the hospital but this discharge could be with the consent of the physician or discharge against medical advices. About $2.5 \%$ of the patients also died. The results of our study showed that there is no significant relationship between treatment outcome and correct triage. As a result, incorrect triage diagnosis cannot be a factor in the change in treatment outcome. However, in the study by Kariman et al, the relationship between treatment outcome and triage level was significant (13). In the study by Esmailian et al, the treatment outcome was $44.1 \%$ hospitalization, $52.6 \%$ discharge, and 3.3\% death, which death percent was higher than our study (14). In the study by Wuerz et al, The relationship between triage level and treatment outcome was not significant and $80 \%$ of patients were alive, $9 \%$ were dead and $11 \%$ were uncertain (7).

\section{Conclusion}

Of the 800 cases reviewed, 107 were not properly triaged (13.3\%). This percentage can be indicative of nurses' inaccurateness at the time of triage or insufficient training of nurses in patient triaging. Of the 107 incorrect triages, 44 were under triage and 63 were over triage. In over triage patients, patients go to a level with higher urgency than what they need, resulting in emergency beds and resources allocated to a patient with less urgency need and under triage can endanger the patient's life over time because the urgency of the patient's treatment needs is underestimated than the actual need. Therefore, proper triage planning, especially in Rasoul Akram hospital, should be a priority in the emergency department.

\section{Limitations of the study}

Access to patient records and corrupted, illegible or unavailable triage sheet (missing) in a number of cases that caused the files to be changed and as a result wasted time.

Authors' contribution

NT and AT designed the study, observed accuracy and validity of the study. AT and SA collected the data and follow the study. AT, NT and SA supervised the project. AT and NT wrote the paper. All authors edited and revised the final manuscript and accepted its publication.

Conflicts of interest

There was no conflict of interest in the current research.

Ethical considerations

Ethical issues (including plagiarism, data fabrication, double publication) have been completely observed by the authors.

\section{Funding/Support}

This study was supported by Iran University of Medical Sciences, Tehran, Iran.

\section{References}

1. Christ M, Grossmann F, Winter D, Bingisser R, Platz E. Modern triage in the emergency department. Dtsch Arztebl Int. 201;107:892-8. doi:10.3238/arztebl.2010.0892.

2. Parenti N, Reggiani ML, lannone P, Percudani D, Dowding D. A systematic review on the validity and reliability of an emergency department triage scale, the Manchester Triage System. Int J Nurs Stud. 2014;51:1062-9. doi: 10.1016/j. ijnurstu.2014.01.013.

3. Göransson KE, von Rosen A. Interrater agreement: a comparison between two emergency department triage scales. Eur J Emerg Med. 2011;18:68-72. doi:10.1097/MEJ.0b013e32833ce4eb

4. Manos D, Petrie DA, Beveridge RC, Walter S, Ducharme J. Inter-observer agreement using the Canadian emergency department triage and acuity scale. CJEM. 2002;4:16-22. doi: 10.1017/s1481803500006023.

5. FitzGerald G, Jelinek GA, Scott D, Gerdtz MF. Emergency department triage revisited. Emerg Med J. 2010;27:86-92. doi: 10.1136/emj.2009.077081.

6. Safari S, Rahmati F, Baratloo A, Motamedi M, Forouzanfar MM, Hashemi B, Majidi A. Hospital and pre-hospital triage systems in disaster and normal conditions; a review article. J Emerg Med. 2015;2:2-10.

7. Wuerz RC, Milne LW, Eitel DR, Travers D, Gilboy N. Reliability and validity of a new five-level triage instrument. Acad Emerg Med. 2000;7:236-42. doi: 10.1111/j.1553-2712.2000. tb01066.x.

8. Göransson K, Ehrenberg A, Marklund B, Ehnfors M. Accuracy and concordance of nurses in emergency department triage. Scand J Caring Sci. 2005;19:432-8. doi: 10.1111/j.14716712.2005.00372.x.

9. $\mathrm{Ng} \mathrm{CJ}, \mathrm{Hsu} \mathrm{KH}$, Kuan JT, Chiu TF, Chen WK, Lin HJ, Bullard MJ, Chen JC. Comparison between Canadian triage and acuity scale and Taiwan triage system in emergency departments. J Formos Med Assoc. 2010;109:828-37.

10. Green NA, Durani Y, Brecher D, DePiero A, Loiselle J, Attia M. Emergency Severity Index version 4: a valid and reliable tool in pediatric emergency department triage. Pediatr Emerg Care. 2012;28:753-7. doi: 10.1097/PEC.0b013e3182621813.

11. Dehnadi Moghadam A, Hemati H, Shabani S. Comparison the number of triaged patients in three working shift in poursina hospital in rasht. J Guil Med. 2008;17:68-76.

12. Kamrani F, Ghaemipour F, Nikravan M, Alavi Majd H. Prevalence of miss triage and outcomes under triage of patients in emergency department. J Heal Manag. 2013;2:17-23. 
13. Kariman H, Joorabian J, Shahrami A, Alimohammadi H, Noori Z, Safari S. Accuracy of emergency severity index of triage in Imam Hossein hospital-Tehran, Iran (2011). J Gorg Med. 2013;15:115-120.

14. Esmailian M, Zamani M, Azadi F, Ghasemi F. Inter-rater agreement of emergency nurses and physicians in Emergency Severity Index (ESI) triage. Emerg (Tehran) 2014;2:158-61.

15. Jafari-Rouhi AH, Sardashti S, Taghizadieh A, Soleimanpour $\mathrm{H}$, Barzegar M. The Emergency Severity Index, version 4, for pediatric triage: a reliability study in Tabriz Children's Hospital, Tabriz, Iran. Int J Emerg Med. 2013;6:36. doi: 10.1186/18651380-6-36.

16. Rahmani F, Sepehri Majd P, Ebrahimi Bakhtavar H, Rahmani F. Evaluating the accuracy of emergency nurses in correct triage using emergency severity index triage in Sina hospital of Tabriz: a cross-sectional analysis. J Trauma Emerg. 2018; 4:9-13.

17. Rutschmann OT, Hugli OW, Marti C, Grosgurin O, Geissbuhler A, Kossovsky M, et al. Reliability of the revised Swiss Emergency Triage Scale: a computer simulation study. Eur J Emerg Med. 2018;25:264-269. doi: 10.1097/MEJ.0000000000000449

18. Savatmongkorngul S, Yuksen C, Suwattanasilp C, Sawanyawisuth K, Sittichanbuncha Y. Is a mobile emergency severity index (ESI) triage better than the paper ESI?.Intern Emerg Med. 2017;12:1273-1277. doi: 10.1007/s11739-0161572-x

19. Mirhaghi A, Kooshiar H, Esmaeili H, Ebrahimi M. Outcomes for emergency severity index triage implementation in the emergency department. J Clin Diagn Res. 2015;9:OC04-7. doi: 10.7860/JCDR/2015/11791.5737.

20. Seyedhosseini-Davarani $S$, Nejati A, Hossein-Nejad $H$, Mousavi SM, Sedaghat M, Arbab M, et al. Outcome-based validity and reliability assessment of raters regarding the admission triage level in the emergency department: a crosssectional study. Adv J Emerg Med. 2018;2:e32. doi: 10.22114/ AJEM.v0i0.76.

21. Ghanbarzehi N, Balouchi A, Sabzevari S, Darban F, Khayat NH. Effect of triage training on concordance of triage level between triage nurses and emergency medical technicians. J Clin Diagn Res. 2016;10:IC05-7. doi: 10.7860/JCDR/2016/20328.7866.

22. Buschhorn HM, Strout TD, Sholl JM, Baumann MR. Emergency medical services triage using the emergency severity index: is it reliable and valid?. J Emerg Nurs. 2013;39:e55-63. doi: 10.1016/j.jen.2011.11.003. 